(Aus dem Staatlichen Hygienischen Institut zu Hamburg. Direktor: Prof. Dr. Dunbar).

\title{
Über die Ansflockungsreaktionen von Sachs-Georgi und Meinicke (D. M.) zur Serodiagnostik der Syphilis。
}

\author{
Von
}

\author{
Prof. Dr. W. Gaehtgens, \\ Vorsteher der serologischen Abteilung.
}

Zahlreiche Untersuchungsmethoden sind im Laufe dor letzten Jahro als Ersatz bzw. Frgänzung der Wassermannschen Reaktion empfohlen worden. Von ihnen beanspruchen die einzeitigen kolloidalen Globulinflockungsroaktionen von Sachs-Georgi (Med. Klinik 1918, S. 805 und Münch. mod. Wochenschr. 1919, S. 242) und Meinicke (Münch. med. Wochensehr. 1918, Nr. 49 und 1919, Nr. 33) besonderes Interesse. Beide zeichnen sich durch die verblüffende Einfachheit der Ausführung ans. die gegenüber der komplizierten Technik der Wassermannuntersuchung als erheblicher fortschritt bezeichnet werden mul3 und demgemäß zu zahlreichon Nachprüfungen Anregung gegeben hat. Bereits vor Jahresfrist habo ich (Müneh. med. Wochenschr. 1919, Nr. 33) über die Ergebnisse berichtet, die ich bei der Untersuchung von 700 Blut- und Iiquorproben nach Sachs-Georgi erhalten hatte. Die damaligen günstigen Erfahrungen veranlaßten mich, das Verfahren auch weiterhin neben - ler Wassermann-Reaktion bei allen Blutuntersuchungen beizubehalten. AuBer der Sachs-Georgi-Reaktion habe ich neuerdings auch die von Meinicke ausgearbeitete sog. dritte Modifikation, deren Einzeitigkeit besonders zugunsten ihrer Einführung in einen größeren Untersuchungsbetrieb sprach, zur Anwendung gebracht. Die bei diesen Untersuchungen gewonnenen Resultate miteinander zu vergleichen und auch zur Wassemann-Reaktion in Bezichung zu setzen, ist der Zweck der vorliegenden Ausführungen.

I.

Über die hinlänglich bekannte Reaktion nach Sachs-Georgi kann ich mich kurz fassen, da diese Untersuchungen meine früheren Beobachtungen im wesentlichen bestätigen.

Die Versuchsanordnung schloß sich ganz an die Vorschriften der sog. Brutschrankmethode an: $1 \mathrm{ecm}$ des inaktivierten, auf. 1. : 10 verdünnten Patienten- 
serums wurde mit 0,5 cem auf $1: 6$ verdünnten Extraktes gemischt, mit den Kontrollen für 24 Stunden in den Brutschrank gestellt und das Resultat am folgenden Tage sowie nach 48 Stunden abgelesen. Als Extrakt diente ein im Institut hergestellter, mit 1 Prom. Cholesterin versetzter alkoholischer Auszug aus normalem Rinderherzen. In meinen früheren Untersuchungen hatte ich bereits diesen Extrakt eingehend mit Sachs-Georgischen Originalextrakten, die mir Herr Prof. Sachs in liebenswürdiger Weise zur Verfügung gestellt hatte, vergleichen und seine Brauchbarkeit feststellen können. Von der. Verwendung mehrerer verschiedenartiger Extrakte, die ich in Übereinstimmung mit anderen Autoren früher selbst empfohlen hatte, mußte ich aus äußeren Gründen Abstand nehmen. Die Untersuchung nach Wassermann erfolgte in Anlehnung an die Originalvorschrift, mit mindestens 2 verschiedenen alkoholischen Extrakten, nämlich einem Auszug aus normalem Rinderherz und einem cholesterinierten Auszug aus syphilitischem Menschenherz. Der Komplementbedarf wurde jedesmal austitriert. Im ganzen wurden 1102 Proben (1093 Sera, 9 Liquorproben) gleichzeitig nach Sachs-Georgi and Wassermann mit folgendem Ergebnis untersucht.

Tabelle I.

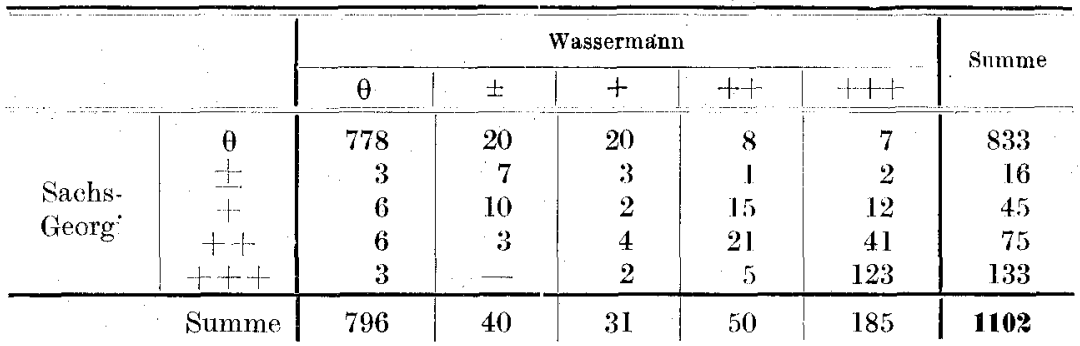

Zeichenerklärung: $\theta=$ negativ $=$ keine Ausflockung bzw. Komplementbindung. $+=$ zweifelhaft $=$ sehr schwache Ausflockung bzw. Komplementbindung.

- schwach positiv $=$ schwache Ausflockung bzw. Kom. plementbildung.

$++=$ positiv $=$ starke Ausflockung bzw. Komplementbindung. $+++=$ stark positiv $=$ sehr starke Ausflockung bzw. komplette Komplementbindung.

Wie aus Tabelle I zu entnehmen ist, ließ sich bei 93I Proben $=84,5 \%$ eine vollkommene Übereinstimmung in qualitativer und quantitativer Hinsicht zwischen der Sachs-Georgi- und Wassermann-Reaktion feststellen. Läßt man die feineren quantitativen Unterschiede außer Betracht, so erhöht sich die $\mathrm{S}$ u m me der übereinstim mend negativen und einwandfrei positiven $(+-+++)$ Ergebnisse auf $1010=91,6 \%$, bzw. auf $1029=93,4 \%$, wenn auch die mit \pm bezeichneten zweifelhaften Resultate mitberücksichtigt werden. Das sind Zahlen, die vollkommen meine früheren Beobachtungen $(91,7 \%$ bzw. $93,7 \%)$ bestätigen und in Übereinstimmung mit den Erfahrungen zahlreicher anderer Autoren für die praktische Brachbarkeit der Sachs-Georgischen Reaktion sprechen. 
von Sachs-Georgi und Meinicke (I. M.) zur Serodiagnostik der Syphilis. 469

Den 1029 übereinstimmenden Ergebnissen sind im ganzen 73 Fälle = $6,6 \%$ gegenüberzustellen, in denen die Resultate der Untersuchung nach Wassermann und Sachs-Georgi auseinandergehen. Bei dieser Berechnung sind nur diejenigen Befunde berücksichtigt, bei denen dem positiven bzw. zweifelhaften Ausfall der einen Reaktion ein absolutes Versagen des anderen Verfahrens gegenübersteht und umgekehrt. Werden auch die, zweifelhaften" Ergebnisse als unsicher und nicht streng beweisend den negativen Ausschlägen zugezählt, so resultiert cine Gesamtdifferenz beider Methoden in 92 Fällen $=8,4 \%$. Bei 18 Patienten fiel die Untersuchung nach $W$ assermann negativ aus, wïhrend gleichzeitig die Sachs-Georgi-Reaktion positiv bzw. zweifelhaft war. Umgekehrt hatte diese in 55 Fällen ein negatives Resultat bei gleichzeitig positivem bzw. zweifelhaftem Wassermann. Bei beiden Gruppen ließ sich ein Überwiegen bestimmter Luesformen oder ein Einfluß der spezifischen Behandlung nicht mit Sicherheit feststellen. Das steht in einem gewissen Widerspruch zu der von anderen Autoren gemachten Erfahrung, die auch ich früher bestätigen konnte, daß bei behandelten Luesfällen die Sachs-Georgi-Reaktion öfter noch positiv ausfällt, wenn die Untersuchung nach Wassermann schon ein negatives Resultat zeitigt. Möglicherweise mag der aus äußeren Gründen erfolgte Verzicht auf die gleichzeitige Verwendung mehrerer verschiedenartiger Extrakte nicht ohne Finfluß auf meine jetzigen Ergebnjsse gewesen sein. Zu denken wäre natürlich auch daran, daß dic Differenz durch die Wigenart des Untersuchungsmateriales bedingt sein könnte. Jedenfalls liegt es mir forn, die vorliegenden Beobachtungen in Gegensat $\%$ zu don Erfahrungen anderer Autoren und meiner eigenen früheren Mittoilung stellen zu wollen, zumal es mir nicht immer möglich war, genaue Angabon: über die Krankheitsform und die Art der Behandlung zu crhalten.

Die im vorigen Abschnitt mitgeteilten Zahlen lassen schon erkennen, daß die Empfindlichkeit bcider Methoden trotz der weitgehenden Übereinstimmung ihrer Ergebnisse gewisse Verschicdentieiten aufweist. Das kommt nicht nur in den Gesamtresultaten, sondern besonders auch bei Berücksichtigung der quantitativen Verhältnisse zum Ausdruck. Am auffäligsten tritt dieses Verhalten in den Zahlen hervor, welche die negativen und stark positiven Resultate wiedergeben. 185 stark positiven Wassermannbefunden stehen nur 133 nach Sachs-Georgi stark positiv reagierende Proben gegenüber, während andererseits bei 796 Seris die Untersuchung nach $W$ a s s e r m a n n, dagegen bei 833 die Prüfung nach S a chs-Ge orgi negativ ausfiel. Für diese nicht unbeträchtlichen Unterschiede lediglich die Verwendung eines einzigen Extraktes verant wortlich zu machen, erscheint mir nicht angängig, angesichts der weitgehenden Ưbereinstimmung meiner früheren und jetzigen Ergebnisse. Vielleicht ist dieses Verhalten teilweise auf den Einfluß der Brutschrank- 
methode zurückzuführen, die, worauf schon S a ch s und G e or g i (Münch. med. Wochensehr. 1920, S. 66) und auch Stilling (Med. Klinik 1920, S. 41) hingewiesen haben, die Empfindlichkeit des Verfahrens etwas herabzusetzen scheint. Sow ohlin qualitativer als a uch in quantitativer Hinsicht stellt sich somit die Wassermann-Reaktion als die empfindlichere Methode dar. Diese a uch von anderen Autoren (Weichardtu. Schrader, Schroeder, Hinzelmann u. a.) festgestellte Überlegenheit $m u ß$ in der Forderung zum Ausdruck kommen, das Verfahren von Sachs u. Georginicht als Ersatz, sondern nur als leistungsfähige Ergänzung neben der Untersuchung nach Wassermann in Anwendung zu bringen.

II.

Etwa gleichzeitig mit Sachs und Georgi hat Meinicke ein auf ähnlichen Grundsätzen beruhendes Verfahren ausgearbeitet, das er unter dem Namen der Lipoidbindungsreaktion in die Serodiagnostik der Syphilis eingeführt hat. Meinicke (Berl. klin. Wochenschr. 1917, S. 613) hatte anfänglich seine Methode in zwei verschiedenen Formen zweizeitig ausgebaut. In der als „Kochsalzmethode" beschriebenen Versuchsanordnung sollten sich die Globuline des Luesserums mit den Extraktlipoiden verbinden und mit ihnen zusammen ausfallen. Hingegen sollte diese Verbindung in der "Wassermethode" in Lösung bleiben. Eine Reihe von Nachprüfungen (s. Blumenthal, Med. Klin. 1919, S. 772) bestätigte im wesentlichen die Angaben Meinickes und stellte die praktische Brauchbarkeit der Reaktion außer Zweifel. Immerhin bedeutete die $Z$ weizeitigkeit, besonders gegenüber der einfacheren Technik der einzeitigen Sachs-Georgi-Reaktion, einen Nachteil, der ihrer weiteren Verbreitung und Anwendung im Wege stand. Diesem Übelstand hat nun Meinicke in der Weise abgeholfen, daß er seine Lipoidbindungsreaktion in Form der sog. ,,dritten Modifikation“ (D. M.) einzeitig gestaltet hat (Münch. med. Wochenschr. 1918, Nr. 49 und 1919, Nr. 33). Das Prinzip der ,dritten Modifikation“ beruht darauf, die positiven syphilitischen Sera bei einem relativ hohen Kochsalzgehalt der Versuchsflüssigkeit zur Ausfällung zu bringen. Die Frage, ob den Vorgängen der ,Lipoidbindungsreaktion" eine Bindung von Extraktlipoiden und Serumglobulinen oder eine Störung des Kochsalzgleichgewichtes der Serumglobuline im Sinne einer Kochsalzentziehung durch die Extraktkolloide zugrunde liegt, läßt Meinicke auf Grund neverer Untersuchungen (Zeitschr. f. Immunitätsforsch. 29, 396. 1920) unentschieden.

Von entscheidender Bedeutung für die Ausführung der dritten Modifikation ist die Verwendung eines brauchbaren Organextraktes. Meinicke empfiehlt dafür Pferdeherzen, die er trocknet, zu einem 
von Sachs-Goorgi und Meinicko (I). M.) zur Serodiagnostik der Syphilis. 471

feinen Pulver zerreibt und dann durch Behandlung mit Äther von störenden, fettigen Beimengungen befreit. Das in dicser Weise vorbereitete Herzpulver wird hierauf mit Alkohol extrahiert und liefert einen Auszug, dessen für die Untersuchung geeignete Konzentration durch besondere Versuche ermittelt werden muß. Tch hatte mir nach diesen Angaben selbst einen alkoholischen Pferdeherzextrakt hergestellt, dessen Brauchbarkeit ich in vergleichenden Untersuchungen mit einem mir in liebenswürdiger Weise von Herrn Dr. Meinic ke zur Verfügung gestellten Originalextrakt feststellen konnte. Von 230 Serumproben. reagierten 213 mit dem Original- und dem Institutsextrakt völlig übercinstimmend. In 14 positiven Fällen war die Ausflockung mit dem Institutsextrakt quantitativ etwas stärker erfolgt als mit dem Originalextrakt, während sich das umgekehrte Verhalten nur einmal feststellen ließ. Endlich ergab in 2 nach Sachs-Goorgi und Wassermann positiven Fällen der Institutsextrakt schwache, der Originalextrakt dagegen keine Fällung. Das Ergebnis dieser Kontrolluntersuchungen kann also dahin zusammengefaß3t werden, da $B$ der von mir hergestellte Pferdeherzauszug sich dem Meinickeschen Originalextrakt als mindestens cbenbürtig, mithin für die praktische Verwendung als geeignet erwiesen hat.

Als zweiten Kontrollextrakt habe ich anfangs einen von der Firma Leitz in Berlin nach don Angaben Lessers (Med. Klin. 1919, S. 822) hergesteliten alkoholischen Menschenherzextrakt benutzt. Die bei der Untersuchung von 113 Serumproben mit diesem Auszug gesammelten Erfahrungen führten indes zu einer weniger günstigen Beurteilung als boim Meinickeschen Originaloxtrakt. Einmal ließen, entsprechend Lessers Beobachtung, 6 Sera in der Flockungskontrolle die Flockung vormissen, so daß die oft wünschenswerte Ergünzung der einzeitigen Molifikation nicht möglich war. Vor allem aber zeigte es sich, daß der Lessersehe Extrakt eincrseits in positiven Fällen eine geringere Empfindlichkeit als der Meinickesche und der im Institut hergestelte Auszug aufwies, andererseits in sicher negativen Fällen recht häufig zweifelhafte Resultate ergab. Diese Erfahrungen veranlaß3ten mich, von der weiteren Verwendung des Extraktes Abstand zu nehmen.

Die Ausfiihrung der Untersuchungen gestaltete sich im Anschluß an die Vorschift M e in ic k es folgendermaßen: Die für die Versuche erforderliche Menge des gebrauchfertigen Wxtraktes wurde mit der halben Menge destillierten Wassers vermengt und dieses Gemisch nach einstündigem Stehen dureh schnelles Hinzufügen der siebenfachen Qnantität 2 proz. Kochsalzlösung weiterverdïnnt. $Z / u$ $0,8 \mathrm{ccm}$ Extraktverdünnung wurden hierauf $0,2 \mathrm{ecm}$ des jeweils zu untersuchenden, $15 \mathrm{Min}$. bei $55-56^{\circ} \mathrm{C}$ inaktivierten Serums hinzugefügt und die Röhrehen $20-24$ Stunden im Brutsehrank gehalten. Die positiven Proben zeigten alsdann im Gegensatz zu den negativen eine mehr odcr weniger stark ausgesprochene Ausflockung. Auf diese Weise wurden im ganzen 1018 Proben, darunter 9 Lumbalpunktate, mit folgendem Ergebnis untersucht. 
Tabelle II.

\begin{tabular}{|c|c|c|c|c|c|c|c|}
\hline & \multicolumn{5}{|c|}{ Wassermann } & \multirow{2}{*}{ Summe } \\
\hline & & $\theta$ & \pm & + & ++ & $+1+$ & \\
\hline \multirow[t]{2}{*}{$\begin{array}{l}\text { Meinicke } \\
\text { (3. Mod.) }\end{array}$} & $\begin{array}{c}\theta \\
+- \\
+ \\
++ \\
+++1 . \\
\end{array}$ & $\begin{array}{r}723 \\
3 \\
3 \\
1 \\
1 \\
\end{array}$ & $\begin{array}{r}20 \\
4 \\
6 \\
5 \\
1 \\
\end{array}$ & $\begin{array}{r}15 \\
3 \\
6 \\
4 \\
2 \\
\end{array}$ & $\begin{array}{r}8 \\
3 \\
14 \\
10 \\
12 \\
\end{array}$ & $\begin{array}{r}8 \\
9 \\
24 \\
133\end{array}$ & $\begin{array}{r}774 \\
13 \\
38 \\
44 \\
149\end{array}$ \\
\hline & Summe & 731 & 36 & 30 & 47 & 174 & 1018 \\
\hline
\end{tabular}

Eine vollkommene Ubereinstimmung in qualitativer und quantitativer Hinsicht ließ sich, wie aus Tabelle II hervorgeht, bei 876 Proben $=$ $86 \%$ feststellen. Werden die einwandfrei positiven $(+-+++)$ Fälle ohne Berücksichtigung der quantitativen Unterschiede als übereinstimmend positiv gerechnet, so erhöht sich diese Zahl a uf $\mathbf{9 4 1}=\mathbf{9 2 , 4} \%$; werden auch die zweifelhaften Befunde $(t)$ als bedingt positive Ergebnisse in Betracht gezogen, wie es oben bei der Sachs-Georgi-Reaktion geschah, so ergibt sich eine Ubereinstimmung in 959 Fällen $=94,2 \%$. Diese Beobachtung bestätigt demnach die Angaben von Meinicke, der Differenzen nur in $5-10 \%$ der Fälle hat feststellen können. Auch Huebschmann (Münch. med. Wochenschr. 1920, S. 251), der, soweit ich der mir zugänglichen Literatur entnehmen konnte, bisher als einziger seine Erfahrungen mit der dritten Modifikation veröffentlicht hat, gelangt zu ähnlichen Ergebnissen.

In 59 Fällen $=5,8 \%$ ließ sich andererseits ein vollständiges Auscinandergehen der Untersuchungsresultate nach Wassermann und Meinicke feststellen. Diese Zahl erhöht sich auf $77=7,6 \%$, wenn auch die ,zweifelhaften ", also nicht streng beweisenden Befunde den negativen Ergebnissen zugezählt werden. Bei 51 Patienten fiel die dritte Modifikation negativ aus, während die Untersuchung nach Wasser mann noch ein positives bzw. zweifelhaftes Resultat zeitigte, Umgekehrt reagierten nur 8 Fälle mit negativem Wassermann nach Meinicke positiv bzw. zweifelhaft. In beiden Gruppen ließ sich ein Überwiegen bestimmter Luesformen oder ein Einfluß der spezifischen Behandlung nicht feststellen. Doch mögen hierfür die schon oben. erörterten Faktoren, ebenso wie bei der Sachs-Georgi-Reaktion, verantwortlich zu machen sein.

Trotzdem die Ergebnisse der Untersuchung nach Wassermann and nach Meinicke eine weitgehende Übereinstimmung zeigen, geht aus den eben mitgeteilten Zahlen doch auch hervor, da B die Empfindlichkeit der Wassermannreaktion diejenige der Lipoidbindungsreaktion übertrifft. Das kommt am auffälligsten in den 
von Sachs-Georgi und Meinicke (D. M.) zur Serndiagnostik der Syphilis. 473

Kahlen zum Ausdruck, welche die negativen und stark positiven Befundo wiedergeben. Hier lassen die 731 negativen und 174 stark positivon Wassermannresultate gegenüber 774 negativen und 149 stark positiven Ergebnissen nach Meinicke die zweifellose Ủberlegenheit der Wassormannreaktion erkennen. Ähnlich wie bei dem Verfahren von Sachs und Georgi muß diese Erkenntnis auch hier zu dem Schluß führen, daß die dritte Modifikation die Wassermannreaktion nicht ersetzen, sondern nur wirkungsvoll ergänzen soll und darf.

TII.

Die in den beiden vorigen Abschnitten wiedergegebenen Zahlen ermöglichen schließlich noch den direkten Vergleich der Sachs-GeorgiReaktion mit der dritten Modifikation, da beide Verfahren bei dem größten Teil der untersuchten Proben gleichzeitig in Anwendung gobracht werden konnten. Bei der Frörterung der Frage, welche von beiden Methoden die leistungsfähigere ist, bin ich mir dessen bewußt, daß ich mir cin endgültiges Urteil darüber versagen muß, da mein Beobachtungsmaterial nicht groß genug ist und die festgestellten Unterschiede nicht so tiefgreifender Art sind, um prinzipielle Bedeutung beanspruchen zu können. Immerhin lassen sich gewisse Differenzen doch zweifellos erkennen.

Die in den vorigen Abschnitten aufgestellten Berechunngen äber clie Ubereinstimmung von Wassermann-und Ausflockungs-Reaktion zeigen in dieser Hinsicht eine leichte Uberlegenheit der dritten Modifikation $(92,4 \%$ bzw. 94,2\%) über die Methode von Sachs-Georgi $(91,6 \%$ bzw. 93,4\%). Deutlicher treten diese an sich geringfügigen Unterschicde in Frscheinumg, wenn die in Tabelle I und II niedergelegten Frgebnisse cimzeln miteinander verglichen werden. Fs zoigt sich alsdann, daß von 174 stark positiven Wassermannbefunden 133 auch nach Meinicke stark positiv bewertet werden konnten, während von 185 stark positiven Wassermannresultaten nur 123 auch die Diagnose stark positiv nach Sachs-Georgi erhielten. Es betrug also die Differenz bei Sachs(icorgi 62 Fälle, bei Meinicke dagegen nur 41. Vergleicht man ferner dic nogativen Frgebnisse, so läßjt sich feststellen, daß von 731 wassermannnegativen Proben 723 negativ und nur 8 positiv bzw. zweifelhafi nach. Meinicke reagierten. Andererseits hatte von 796 wassermannnegativen Proben die Untersuchung nach Sachs-Georgi bei 778 ein übereinstimmend negatives, bei 18 dagegen ein positives bzw. zweifelhaftes Ergebnis. Das sind Unterschiede, die nicht so geringfügiger Art sind, um nur als zufällig angesprochen werden zu können. Vielmehr deuten sie auf eine leichte Überlegenheit der dritten Modifikation hin, soweit die Úbereinstimmung mit der Wassermannreaktion in Frage kommt. Dieser Nachteil der Sachs-Georgi- 
schen Methode wird aber dadurch nahezu wiederausgeglichen, daß die Zahl der positiven Befunde mittlerer Stärke $(+$ und $++)$ hier größer ist als bei der dritten Modifikation und infolgedessen die durchschnittliche Leistungsfähigkeit des Verfahrens nicht wesentlich hinter derjenigen der Lipoidbindungsreaktion zurückbleibt. Als direkter Vorzug der Sachs-Georgischen Reaktion ist außerdem der Umstand anzusprechen, daß sie in einer nicht unbeträchtlich größeren Anzahl ron wassermannnegativen Fällen noch ein positives Resultat zu zeitigen vermag.

Eine Ergänzung des eben Gesagten bringt die Tabelle III, welche die Einzelergebnisse der Untersuchungen nach Sachs-Georgi und Meinicke in Beziehung zueinander bringt.

Tabelle III.

\begin{tabular}{|c|c|c|c|c|c|c|c|}
\hline & & \multicolumn{5}{|c|}{ Sachs-Georgi } & \multirow{2}{*}{ Summe } \\
\hline & & $\theta$ & \pm & + & +1 & +++ & \\
\hline $\begin{array}{l}\text { Meinicke } \\
\text { (3. Mod.) }\end{array}$ & $\begin{array}{c}\theta \\
+ \\
+ \\
++ \\
++1 \\
+\end{array}$ & $\begin{array}{r}751 \\
2 \\
6 \\
4 \\
4 \\
\end{array}$ & $\begin{array}{r}5 \\
4 \\
3 \\
1 \\
-\end{array}$ & $\begin{array}{r}7 \\
3 \\
16 \\
12 \\
3 \\
\end{array}$ & $\begin{array}{r}5 \\
3 \\
13 \\
17 \\
33 \\
\end{array}$ & $\begin{array}{r}5 \\
-2 \\
8 \\
108\end{array}$ & $\begin{array}{r}773 \\
12 \\
40 \\
42 \\
148\end{array}$ \\
\hline & Summe & 767 & 13 & 41 & 71 & 123 & 1015 \\
\hline
\end{tabular}

Wie aus Tabelle III hervorgeht, reagierten von 1015 gleichzeitig nach Sachs-Georgi und Meinicke untersuchten Proben in quantitativer und qualitativer Hinsicht völlig übereinstimmend $896=88 \%$. Werden die quantitativen Unterschiede außer acht gelässen, so erhöht sich die Zahl der übereinstimmend negativen und positiven Fälle $(+-+++)$ a uf $967=95 \%$, bzw. auf $977=96 \%$, wenn auch die zweifelhaften Ergebnisse ( \pm ) mitberücksichtigt werden. Angesichts dieser weitgehenden Übereinstimmung läßt sich von einer ausgesprochenen Überlegenheit der einen Reaktion über die andere nicht reden. Erst bei Würdigung der Einzelergebnisse treten gewisse Unterschiede deutlicher hervor. Die Tatsache, daß 148 stark positiven Befunden nach Meinicke nur 123 stark positive nach Sachs-Georgi gegenüberstehen, spricht entschieden zugunsten einer etwas größeren Empfindlichkeit der dritten Modifikation in quantitativer Hinsicht. Der Unterschied zwischen schwacher und starker Ausflockung ist hier ausgeprägter, als bei der Sachs-Georgireaktion, bei welcher der Übergang von starken zu schwachen Ausfällungen allmählicher erfolgt. Reaktionenmittleren Grades $(++)$ werden bei dem Verfahren nach Sachs-Georgi öfter ange- 
von Saclıs-Georgi und Meinieke (D. M.) zur Serodiagnostik der Syphilis. 475

troffen als bej der dritten Modifikation, die ihrerseits wieder: durch das häuligere Vorkommen von maximaler Flockenbild ung a usgezeichnet ist. Andererseits kommt die oben erwähnte höhere qualitative Empfindlichkeit der Sachs-Georgi-Reaktion in der allerdings nur wenig niedrigeren Anzahl von negativen Ergebnissen zum Ausdruck. Immerhin sind diese Differenzen so geringfügiger Art, daß von einer a usgesprochenen Überlegenheit des einen oder des anderen Verfahrens nicht die Rede sein kann. Für die Praxis könnte demnach die Anwendung einer Methode als genügend erachtet werlen, wenn nicht, was am meisten zu empfehlen, zur gegenseitigen Ergänzung die gleichzeitige Ausführung beider Untersuchungen möglich ist.

Gewisse Vorzüge und Nachteile ergeben sich für beide Reaktionen auch bei Erörterung der Extraktfrage, die für das Endresultat von entscheidender Bedeutung ist. In beiden Fällen ist die richtige Bereitung und Einstellung des Organauszuges von allergrößter Wichtigkoit. Während sich mir bei der Verdünnung des Pferdeherzextraktes für die dritte Modifikation niemals Schwierigkeiten crgeben haben, konnte ich bei dem Sachs-Georgischen Extrakt in Bestätigung meiner früheren Angaben wieder feststellen, daß zu schnelles Verdünnen von ebenso schädlicher Wirkung wie zu langsames sein kann. In dcm einen Falle ist der Verlust, in dem anderen eine zu hohe Steigerung der Empfindlichkeit die unerwünschte Folge falscher Manipulationen. Am zweckmäßigsten hat es sich mir crwiesen, beim Verdünnen so vorzugehen, dab die erforderliche Gesamtmenge von physiologischer Kochsalzlösung in Einxelportionen von je 1 cem zum Extrakt zugesetzt und durch 3-4malige, in horizontaler Bichtung orfolgende Bewogungen mit diesem gemischt wird. Als Nachteil des Meinickeschen Txtraktes mul3 demgegenüber bezeichnet werlen, daß er nicht wie der Sachs-Georgische gleich nach dem Verlünnen gebrauchsfertig ist, sondern nach flom Wasscrzusatz noch eine Stunde stehen mul3, bevor er weiter verdünt werden kann.

Daß im übrigen der Meinicke-Extrakt sich zur Liquoruntersuchung ebenso gat wie der Sachs-Georgi-Extrakt eignet, konnte ich an dex allerdings nur kleinen 7ahl von 9 Lumbalpunktaten feststellen. Von diesen reagierten übereinstimmend nach Wassermann, SachsGeorgi und Meinicke 4 positiv und 5 nogativ.

Zue Frage der Spezifität beider 'Fällungsreaktionen Stellung zu nehmen, ist mir bei der Art meines Untersuchungsmaterials nur in beschränktem Maße möglich. Soweit ich Gelegenheit hatte, meine Hrgebnisse mit den klinischen Erscheinungen in Beziehung zu bringen, konnte ich feststellen, daß positive Reaktionen sowohl nach SachsGeorgi als auch nach Meinicke nur bei sicheren, teils behandelten, teils unbehandelten Luesfüllen und bei sicher Luesverdachtigen auf- 
getreten waren. Bei über 50 gesunden Personen, 23 Gonorrhoikern, 7 Tuberkulosekranken, ferner bei einer Anzahl von Erkrankungen an Ulcus molle, Rhachitis usw. fiel die Untersuchung sowohl nach SachsGeorgi als auch Meinicke in Übereinstimmung mit der Wassermannreaktion immer negativ aus.

Für die Ergebnisse der Ausflockungsreaktionen ist schließlich auch die Versuchsdauer von Bedeutung. Sowohl die dritte Modifikation von Meinicke als auch die Brutschrankmethode von Sachs-Georgi sieht die Ablesung der Resultate vor, nachdem die Proben 18-24 Stunden bei $37^{\circ} \mathrm{C}$ gestanden haben. Darüber hinaus habe ich, zumal bei zweifelhaften und schwachpositiven Ergebnissen, nochmals einen 24stündigen Brutschrankaufenthalt angeschlossen und dadurch gar nicht selten die wünschenswerte Verstärkung der Ausflockung erhalten. Unspezifische Fällungen, die nach 24 Stunden auftraten, nach 48 Stunden aber wieder verschwunden sind, habe ich nur bei Anwendung des Originalverfahrens von Sachs und Georgi (2 Stunden Brutschrank-, 18 Stunden Zimmertemperatur) früher wiederholt festgestellt. Bei der ,Brutschrankmethode" habe ich hingegen diese Erscheinung, die inzwischen durch Stilling (Med. Klinik 1920, S. 41), Frommherz, Pöhlmann und Baumgärtel (s. Baumgärtel, Münch. med. Wochenschr. 1920, S. 421) bestätigt worden ist, in dem Grade nicht wieder beobachten können. Wenn also auch die Brutschrankmethode eine etwas geringere Empfindlichkeit der Reaktion zur Folge haben mag, so bedingt sie andererseits doch wieder den unzweifelhaften Vorteil, daß derartige unspezifische Ausflockungen vermieden bzw. a uf ein Minimum reduziert werden. Auch bei der dritten Modifikation habe ich diese reversible Körnchenbildung nicht festgestellt.

Eine wesentliche Verkürzung der Versuchsdauer läßt sich durch Anwendung des von mir (Münch. med. Wochenschr. 1906, S. 1351) ursprünglich für die Bakterienagglutination empfohlenen Zentrifugierverfahrens erreichen. Dafür ist es nicht notwendig, die Extrakt-Serumgemische zuerst 3-4 Stunden lang der Brutschranktemperatur auszusetzen, wie es K. Meyer (Med. Klinik 1919, S. 262) empfohlen hat. Es genügt vielmehr, wie ich schon in meiner ersten Arbeit mitgeteilt habe, die Röhrehen gleich nach der Mischung ca. 20 Minuten lang kräftig auszuschleudern, um das Ergebnis zu erhalten. Ebenso wie bei der Sachs-Georgischen Methode gelingt es auch bei der dritten Modifikation von Meinicke, den Ablauf der Reaktion in dieser Weise zu beschleunigen. Nach halbstündigem Ausschleudern zeigen sich auch hier die negativen Proben gänzlich unverändert. In stark positiven Seris ist dagegen am Boden des Gläschens (mit runder Kuppe) ein unregelmäßig gestaltetes, zartes Sediment wahrnehmbar, das sich nach mehrmaligem leichtem Schütteln in kleine, aber deutlich erkennbare 
Flocken auflöst. In schwach positiven und zweifelhaften Fällen ist der Bodensatz schwächer ausgebildet und beschränkt sich mitunter nur auf zarte, strich- und punktförmige Niederschläge. Nach dem Schütteln lassen sich aber auch hier die Flöckchen einwandfrei erkennen.

Im. Anschluß an diese Versuche sei noch kurz über einige gemeinsam mit Herrn Dr. J. Freund-Budapest ausgeführte Untersuchungen herichtet, die sich mit der Frage beschäftigten, in welcher Weise physikalische Finflüsse anderer Art auf den Ablauf der Ausflockungsreaktionen einwirkten. Zu diesem Zwecke wurden die Extraktserumgemenge teils gleich nach der Mischung, teils auch erst nach 3 stündigem Aufenthalt im Brutschrank in den Schüttelapparat gebracht und hier $1 / 2$ Stunde lang kräftig geschüttelt. Es zeigte sich, daß die gleich nach dem Ansetzen mit Meinickeextrakt geschüttelten Proben durch die Bewegung im wesentlichen unverändert geblieben waren; nur einmal liel sich in einem auch nach Wassermann und Sachs-Georgi stark positiven Serum eine dentliche Ausflockung feststellen. Von den zuerst 3 Stunden lang bebrüteten. Proben zeigte ein Teil schon nach dieser \%eit eine recht starke Fällung, die durch das Schütteln weiter verstärkt wurde. Nur in einem Falle war erst unter dem Finfluß des Sehüttelns eine schwach positive Reaktion aufgetreten, die durch das positive Resultat der Untersuchung nach Wassermann und Sachs-Ceorgi bestätigt wurde. Das Sch ütteln hatte also bei der dritten Modifikation die Ausfockung zwar begünstigt und verstärkt. den Ablauf der Reaktion abor nichtausgesprochenabkïrzon können.

Wesentlich anders gestaltete sich das Bild bei der Sachs-GeorgiReaktion. Hier war in ungefähr der Hälfte der Fälle nach dem Schüttelı sino mehr oler weniger starke Flockenbildung aufgetreten. Wurden mun dio geschüttelten Gläschen in den Brutsehrank gestellt und, wir ïblich, das Ergebuis am folgenden Tage festgestellt, so zeigte ss sich, (lak nicht in allen Proben die Körnchen erhalten geblieben waren. Vielmohr war in einem Teil der Fille die Ausflockung restlos zurückgegangen, und zwar gerade bei denjenigen Sera, die auch nach Wasserma 1 n negativ reagierton. Jagegen ließ sich bei den nach 24 Stunden positiven Proben auch oin positivor Ausfall der Wassermannreaktion ausnahmslos feststellen. Einige wenige Sera sehließlich, die nach dem Schütteln negativ reagiert hatten, zeigten nach 24 Stunden einen positiven Aussehlag in Úbereinstimmung mit der Untersuchung nach Wassermann. Das Schütteln hatte alsoteils eine Beschleunigung der Sachs-Georgi-Reaktion, teils aber auch eine unsperifische Ausflockungzur Folge gehabt. Wenn auch demnach eine Abkürzung der Versuchsdauer auf diesem Wege für die Praxis der Ausflockungsreaktion nicht in Frage kommen kann, so ist es andererseits 
478 W. Gaehtgens: Über die Ausflockungsreaktionen von Sachs-Georgi usw.

theoretisch nicht ohne Interesse, daß derartige unspezifische, in der Wärme reversible. Flockenbildungen ebenso wie durch Temperaturerniedrigung (Neukirch, zit. nach Stilling) auch durch dic Bewegung im Schüttelapparat ausgelöst werden können.

\section{Zusammenfassung.}

Die Ausflockungsreaktion von Sachs-Georgi (Brutschrankmethode) und die dritte Modifikation der Lipoidbindungsreaktion von Meinicke zeichnen sich durch große Einfachheit der Ausführung und eine weitgehende Spezifität für Syphilis aus. Beide Methoden sind etwas weniger empfindlich als die Wassermannreaktion; sie können. diese demnach nicht ersetzen, sollten aber als wertvolle Ergänzung und Verschärfung neben ihr regelmäßig zur Anwendung kommen. Werden beide Ausflockungsreaktionen miteinander verglichen, so ergibt sich eine weitgehende Übereinstimmung ihrer Frgebnisse $(95 \%)$; von einer wesentlichen Ủberlegenheit des einen oder anderen Verfahrens kann nicht gesprochen werden. Bei Berücksichtigung der quantitativen VerhäItnisse läßt sich indes eine etwas größere Empfindlichkeit der Meinickereaktion, die durch das häufigere Vorkommen von maximaler Flockenbildung ausgezeichnet ist, feststellen. Andererseits weist die SachsGeorgi-Reaktion eine etwas höhere Leistungsfähigkeit in qualitativer Hinsicht auf und liefert häufiger als die dritte Modifikation ein positives Resultat bei Fällen, die nach Wassermann negativ reagieren. Gegenüber der vorgeschriebenen 24 stündigen Beobachtungszeit hat die 48 stündige den Vorteil, daß schwach positive und zweifelhafte Ergebnisse meist deutlich verstärkt werden. Eine wesentliche Abkürzung der Versuchsdauer läßt sich bei beiden Reaktionen durch halbstündiges Zentrifugieren erzielen. Halbstündiges Schütteln begünstigt und verstärkt zwar die Ausflockung bei der dritten Modifikation, vermag aber im allgemeinen nicht, den Ablauf der Reaktion abzukürzen. Bei der Sachs-Georgi-Reaktion hat das Schütteln in vielen Fällen eine Beschleunigung zur Folge, in anderen Fällen aber eine unspezifische, in der Wärme reversible Ausflockung, wie sie auch durch Temperaturerniedrigung (Neukirch) ausgelöst werden kann. 